Public Health Service, Geleen, Netherlands; ${ }^{7}$ National Institute for Public Health and the Environment, University Medical Centre, Utrecht, Netherlands

Background The Chlamydia Screening Implementation (CSI) is a Dutch large-scale pilot of an internet-based self-sampling Chlamydia trachomatis $(\mathrm{Ct})$ screening program for 16-29-year-old men and women. The effectiveness of CSI can be estimated from changes in the positivity rate of the sampled individuals, but shifts in healthcare use and CSI participation rates makes modelling a valuable alternative approach for estimating screening effectiveness.

Methods We simulated the spread of $\mathrm{Ct}$ in a heterosexual population of age 13-65, using sexual survey data to parametrise a dynamic sexual contact network. A screening program was implemented in the model by constructing "participation trees", which capture the likelihood to participate given an individuals participation history, as observed in the CSI program. Currently available health-care options to test for and treat $\mathrm{C}$ t were also implemented in the model as a baseline (including trends in their usage), against which the effect of screening could be compared. In order to estimate the longterm effects of screening on the Ct prevalence in the Netherlands, future participation rates were estimated from trends in the yearly number of new participants, and by extrapolation of the participation trees.

Results Compared to a baseline scenario, there is a moderate additional effect of 3 years of screening: the estimated Ct prevalence for the target population (16-29) dropped from $2.8 \%$ to $1.7 \%$ in large cities, and from $1.9 \%$ to $1.2 \%$ in more rural regions. As repeated invitees were likely not to participate, the largest effect of screening occurred in its first year when everyone in the target population was invited for the first time. After 3 years, the largest effect of screening on the $\mathrm{Ct}$ prevalence had been reached. Due to the anticipated further decrease in participation rates the long-term decrease in $\mathrm{Ct}$ prevalence is estimated to be in the range of $0.5-0.7$ and $0.4-0.6$ per cent-points in urban and rural regions, respectively.

Conclusions A continued population based screening program has a permanent additional effect on lowering the $\mathrm{Ct}$ prevalence in the Netherlands, but the size of this effect is strongly tied to the participation rate in the targeted population. Therefore, the accuracy of long-term predictions of screening effectiveness depends on a good model implementation of the available data on participation behaviour.

\section{1-S09.05 DECLINE IN HIV PREVALENCE AMONG YOUNG PEOPLE IN THE GENERAL POPULATION OF COTONOU, BENIN, 1998-2008}

doi:10.1136/sextrans-2011-050109.53

${ }^{1} \mathrm{~L}$ Behanzin, ${ }^{2} \mathrm{~A}$ Buve, ${ }^{3} \mathrm{C} \mathrm{M}$ Lowndes, ${ }^{4} \mathrm{D} \mathrm{M}$ Zannou, ${ }^{5} \mathrm{M}$ Minani, ${ }^{6} \mathrm{~S}$ Anagonou, ${ }^{7} \mathrm{M}$ C Boily, ${ }^{8} \mathrm{~A}$ C Labbe, ${ }^{9} \mathrm{R}$ Bitera, ${ }^{1} \mathrm{M}$ Alary. ${ }^{1}$ Centre hospitalier affilié universitaire de Québec, Quebec, Canada; ${ }^{2}$ Institute of Tropical Medicine, Antwerp, Belgium; ${ }^{3}$ Health Protection Agency, London, UK; ${ }^{4}$ Faculté des Sciences de la Santé de Cotonou, Université d'Abomey-Calavi, Cotonou, Bénin Benin; ${ }^{5}$ Dispensaire IST, Cotonou, Bénin;
${ }^{6}$ Faculté des Sciences de la Santé, Université d'Abomey-Calavi, Cotonou, Bénin; ${ }^{7}$ Imperial College, London, UK; ${ }^{8}$ Hôpital Maisonneuve-Rosemont, Montréal, Canada; ${ }^{9}$ Centre hospitalier affilié universitaire de Québec, Quebec, Canada

Objective Comparative study of the prevalence of HIV and sexually transmitted infections (STI), and associated behaviours in the general population of Cotonou between 1998 and 2008.

Methods In Cotonou, two studies employing similar methods were carried out in 1998 and 2008 respectively. In these studies, the census areas (clusters) were sampled with probability proportional to size. After enumeration of all households in the selected clusters, a certain number of households were randomly sampled from each selected census area (950 in 1998, 1070 in 2008). Consenting adults, aged 15-49 years (but 15-64 years for the men in 2008) were interviewed and screened for HIV, syphilis, and HSV-2 (serologic detection of antibodies for the latter infections), Neisseria gonorrhoeae and Chlamydia trachomatis (nucleic acid amplification assays on urogenital samples). The Roa-Scott $\chi^{2}$ was used to consider the cluster effect in the univariate comparison of proportions. Logistic regression (taking into account the cluster effect) was used for multivariate analysis, adjusting for socio-demographic variables.

Results The global HIV prevalence was stable (3.4\% in 1998 vs 3.1\% in 2008). There was however a trend towards decreasing among men (Abstract O1-S09.05 table 1). The decrease was highly significant among men aged less than 30 (3.0\% in 1998 vs $0.5 \%$ in 2008 , $\mathrm{p}<0.0001)$. A trend towards decreasing prevalence was also observed among women aged less than 20 (2.4\% in 1998 vs $0.5 \%$ in 2008 , $\mathrm{p}=0.102)$. On the other hand, an upward trend was observed among women aged $20+$ (3.8 in 1998 vs $4.8 \%$ in 2008, $p=0.346$ ). Syphilis prevalence also decreased significantly, but this decline was more pronounced among women (Abstract O1-S09.05 table 1). The prevalence of gonorrhoea trended lower among men while prevalence of HSV-2 increased among both men and women (Abstract O1-S09.05 table 1). The proportion of adults who reported condom use during their last extramarital sexual intercourse increased $(23.0 \%$ in 1998 vs $40.1 \%$ in 2008, $\mathrm{p}<0.0001$ ).

Discussion The decrease in HIV prevalence among young people could be explained by the increase in condom use and may also be related to the impact of intensive interventions targeting the prostitution milieu during the same period. The upward trend among older women could be related to a large increase in access to antiretroviral therapy that occurred from 2004 onwards.

\section{1-S09.06 ASSESSING THE IMPACT OF A FSW TARGETED HIV INTERVENTION PROGRAMME ON INCIDENCE AND PREVALENCE IN COTONOU, BENIN}

doi:10.1136/sextrans-2011-050109.54

${ }^{1} \mathrm{~J}$ Williams, ${ }^{1} \mathrm{M}$ C Boily, ${ }^{2} \mathrm{C}$ Lowndes, ${ }^{3} \mathrm{~S}$ Camden, ${ }^{3} \mathrm{E}$ Demers, ${ }^{4} \mathrm{I}$ Minani, ${ }^{4} \mathrm{M}$ Zannou, ${ }^{5} \mathrm{~S}$ Anagonou, ${ }^{6} \mathrm{~A} \mathrm{C}$ Labbé, ${ }^{7} \mathrm{M}$ Alary. ${ }^{1}$ Imperial College London, London, UK; ${ }^{2}$ Health Protection Agency, London, UK; ${ }^{3}$ Centre Hospitalier Affilié Universitaire de Québec CHA, Quebec City, Canada; ${ }^{4}$ Dispensaire IST, Cotonou, Benin; ${ }^{5}$ Centre National

Abstract 01-S09.05 Table 1 Multivariate comparison of HIV/STI prevalence between 1998 and 2008 among men and women of the general population of Cotonou aged 15-49

\begin{tabular}{|c|c|c|c|c|c|c|c|c|c|}
\hline & \multicolumn{3}{|l|}{ Women } & \multicolumn{3}{|l|}{ Men } & \multicolumn{3}{|l|}{ Overall } \\
\hline & $\begin{array}{l}1998 \\
(N=1093)\end{array}$ & $\begin{array}{l}2008 \\
(N=1348)\end{array}$ & p Value* & $\begin{array}{l}1998 \\
(N=1019)\end{array}$ & $\begin{array}{l}2008 \\
(N=1159)\end{array}$ & p Value & $\begin{array}{l}1998 \\
(N=2112)\end{array}$ & $\begin{array}{l}2008 \\
(N=2507)\end{array}$ & p Value \\
\hline HIV & $35(3.5 \%)$ & $50(4.0 \%)$ & 0.3463 & $31(3.4 \%)$ & $21(2.0 \%)$ & 0.2385 & $66(3.4 \%)$ & $71(3.1 \%)$ & 0.9259 \\
\hline$N$ gonorrheae & $9(0.9 \%)$ & $10(0.8 \%)$ & 0.9292 & 10 (1.1\%) & $3(0.3 \%)$ & 0.2464 & $19(1.0 \%)$ & $13(0.6 \%)$ & 0.5770 \\
\hline C trachomatis & $13(1.3 \%)$ & $27(2.2 \%)$ & 0.0834 & $21(2.5 \%)$ & $23(2.2 \%)$ & 0.7977 & $34(1.8 \%)$ & $50(2.2 \%)$ & 0.2337 \\
\hline$T$ pallidum & $12(1.3 \%)$ & $4(0.3 \%)$ & 0.0263 & $16(1.8 \%)$ & $9(0.9 \%)$ & 0.0986 & $28(1.5 \%)$ & $13(0.6 \%)$ & 0.0050 \\
\hline HSV-2 & $275(29.5 \%)$ & 397 (33.2\%) & 0.2124 & 103 (11.9\%) & $181(18.1 \%)$ & $<0.0001$ & $378(21.1 \%)$ & 578 (26.4\%) & 0.0026 \\
\hline
\end{tabular}

${ }^{*} p$ Value from the logistic regression analysis taking into account the cluster effect and adjusting for sex (overall analysis only), age, marital status and education level. 